\title{
Noncompact Fuchsian and quasi-Fuchsian surfaces in hyperbolic 3-manifolds
}

\author{
COLIN Adams
}

\begin{abstract}
Given a noncompact quasi-Fuchsian surface in a finite volume hyperbolic 3-manifold, we introduce a new invariant called the cusp thickness, that measures how far the surface is from being totally geodesic. We relate this new invariant to the width of a surface, which allows us to extend and generalize results known for totally geodesic surfaces. We also show that checkerboard surfaces provide examples of such surfaces in alternating knot complements and give examples of how the bounds apply to particular classes of knots. We then utilize the results to generate closed immersed essential surfaces.
\end{abstract}

57M50; $20 \mathrm{H} 10$

\section{Introduction}

Let $M$ be a closed 3-manifold such that the interior of $\mathrm{M}$ is hyperbolic of finite volume. Then $M$ can be realized as the quotient of hyperbolic 3-space $H^{3}$ by a discrete group of isometries $\Gamma$. As is well known, the boundary of $M$ must be a finite collection of tori. A closed neighborhood of a torus boundary that lifts to a collection of horoballs in $H^{3}$ with disjoint interiors is called a cusp. If a single cusp is expanded until its horoball covers first become tangent in $H^{3}$, we call it a maximal cusp. When there are multiple cusps present, we will often start with disjoint cusps and then expand them until they either touch themselves or each other. A set of cusps such that no additional expansion is possible is called a maximal set of cusps. Typically, we will use the upper-half-space model of $H^{3}$ without additional comment.

Let $\mathrm{S}$ be an essential surface with boundary properly embedded in M. Work of Thurston [16] (see also Bonahon [4] and Canary, Epstein and Green [5]) implies there are three possibilities:

(1) $S$ is a virtual fiber, meaning that a finite cover of the manifold is fibered and the surface is covered by a fiber.

(2) $S$ is accidental, meaning there is a simple closed curve on $S$ that is not parallel to the boundary of $S$ but that can be homotoped to the boundary of $M$. 
(3) $S$ is quasi-Fuchsian.

A surface is quasi-Fuchsian if it is covered by a topological plane in $H^{3}$ such that its limit set on the boundary of $\mathrm{H}^{3}$ is a topological circle bounding two disks. Unlike the classical definition, we do not assume that the two disks bounded by the circle are not interchanged by isometries corresponding to the fundamental group of the surface, to allow for nonorientable surfaces. In the case that the surface lifts to a collection of totally geodesic planes, the surface is said to be Fuchsian or totally geodesic.

In the case that $M$ is a nonfibered hyperbolic knot exterior in the 3-sphere, a minimal genus Seifert surface must be quasi-Fuchsian (see Fenley [9] or Cooper-Long [6] for a second proof.) In Adams-Schoenfeld [2], examples are given of Fuchsian Seifert surfaces for hyperbolic knot and link complements in the 3-sphere.

For an alternating knot exterior, any Seifert surface obtained by applying Seifert's algorithm to a reduced alternating projection is known to be minimal genus (cf Crowell [8], Murasugi [13] or Gabai [11]). Thus, if the knot is not fibered, the surface must be quasi-Fuchsian. (In the case of a special alternating knot, where one of the checkerboard surfaces is orientable, we will prove that this particular Seifert surface cannot be a virtual fiber, even if the knot exterior is fibered.)

Definition 1.1 Let $S$ be a properly embedded surface with boundary in a closed 3manifold $M$ with hyperbolic interior of finite volume. Then $S$ is said to be free if $M-$ $N(S)$ is a handlebody. We say $S$ is totally knotted if $M-N(S)$ has incompressible boundary. We say $S$ is semifree if there exists a compressing disc for $\partial(M-N(S))$. Note that free implies semifree.

Definition 1.2 Given a nontrivial, closed curve $\gamma$ on the boundary of a fixed cusp $C$, the length of the shortest path on $\partial C$ that begins and ends on a minimal representative of $\gamma$, but that is not isotopic into $\gamma$ is called the width of $\gamma$, sometimes denoted $w_{\gamma}$. Note that $w_{\gamma}=\operatorname{area}(\partial C) /|\gamma|$.

The width of a surface with one boundary component is the width of its boundary component, usually in a maximal cusp corresponding to that boundary component. By the width of a hyperbolic knot $K$, denoted $w(K)$, we mean the width of the longitude of $K$ in a maximal cusp, a Seifert surface being the implied surface.

In the case of a surface that has more than one boundary, but at most one boundary per cusp, the relative size of the cusps can be chosen so that the width of each boundary component is identical. The cusps can then be expanded while keeping this true until two such cusps touch or one such cusp touches itself. The corresponding width is called the balanced width of the surface. 
When a surface has more than one boundary component in a given cusp, we must be more careful. That case is addressed in Section 3.

Given a boundary curve $\gamma$ of a properly embedded quasi-Fuchsian surface $S$ corresponding to a cusp $C$ of the manifold $M$ with hyperbolic interior, lift to $H^{3}$ such that $C$ lifts to a horoball centered at $\infty$. A topological plane $P$ covering $S$ has a limit point at $\infty$. The limit set of $P$ is a quasi-line in the xy-plane. Let $P_{1}$ and $P_{2}$ be two vertical planes such that they sandwich the limit set $L(P)$ between them and the Euclidean distance between their boundary lines in the xy-plane is as small as possible. Note that their boundary lines will be parallel.

Definition 1.3 The cusp thickness of $\gamma$ is defined to be the distance between $P_{1}$ and $P_{2}$ as measured in the horizontal plane covering the boundary of the cusp $C$. Typically, $C$ will be chosen to be maximal, or to be in a balanced collection of cusps, or to be in a maximal collection of cusps.

If $S$ has one boundary component $\gamma$, the cusp thickness of $S$, denoted $\operatorname{ct}(S)$ is the cusp thickness of $\gamma$. In the case $S$ has more than one boundary component, the cusp thickness of $S$ is defined to be the maximum of the cusp thicknesses of the boundary components.

That there exists such a pair of sandwiching planes follows from the fact that the quotient of the xy-plane by the subgroup of $\Gamma$ that fixes $\infty$ is a torus, and the quasi-line projects to a closed curve on that torus which is homotopic to a geodesic curve in the Euclidean metric inherited by the torus. This implies that the quasi-line must remain a bounded distance from the Euclidean line in the xy-plane that is the projection of the cover of the geodesic.

Definition 1.4 Let $S$ be an incompressible boundary incompressible semifree surface with boundary properly embedded in a 3-manifold $M$ and let $D$ be a compressing disc for $\partial(M-N(S))$. Since $S$ is itself incompressible and boundary incompressible, $\partial D$ alternates between $n$ arcs in $S$ and $n$ arcs in the cusp boundaries for some $n>1$. If $n$ cannot be reduced through isotopy while preserving the fact that $\partial D$ lies in $\partial(M-N(S))$, we say that $D$ is an essential $n-$ gon in the complement of $S$.

The results appearing in the following theorem were proved in Adams et al [1].

Theorem 1.5 Let $S$ be a totally geodesic surface properly embedded in a closed 3-manifold $M$, the interior of which is a cusped hyperbolic 3-manifold, such that $S$ has one or more boundary components, with at most one per cusp. Let $w$ be the balanced width.

Algebraic 83 Geometric Topology, Volume 7 (2007) 
(1) $w \geq 1$.

(2) $w=1$ if and only if there exists an essential 3-gon.

(3) If $S$ is semifree, then $w<2$.

Here, we prove the following additional facts about totally geodesic surfaces.

Theorem 1.6 Let $S$ be a totally geodesic surface properly embedded in a closed 3-manifold $M$, the interior of which is a cusped hyperbolic 3-manifold, such that $S$ has one or more boundary components, with at most one per cusp. Let $w$ be the balanced width.

(1) S possesses no essential 2-gons.

(2) If $S$ possesses an essential $n$-gon for $n \geq 3$, then $w \leq 2 \cos (\pi / n)$.

(3) Let $q$ be the length of the shortest nontrivial curve in the cusp boundary that is not the boundary of $S$. Then $q \geq \sqrt{2}$. If $q=\sqrt{2}$, there exists an essential 3-gon or 4-gon.

Note that Theorem 1.6(2) implies Theorem 1.5(3). As totally geodesic surfaces are special cases of quasi-Fuchsian surfaces, one would hope to have versions of these results that apply to this more general category. However, one must use the cusp thickness invariant to keep track of how far the quasi-Fuchsian surface is from being totally geodesic.

Theorem 1.7 Let $S$ be a quasi-Fuchsian surface properly embedded in a closed 3manifold $M$, the interior of which is a cusped hyperbolic 3-manifold, such that $S$ has one or more boundary components, with at most one per cusp. Let $w$ be the balanced width and ct the cusp thickness corresponding to the cusps giving the balanced width.

(1) $w+c t \geq 1$

(2) If $w+c t=1$, then $S$ possesses an essential 3-gon.

(3) If $S$ is semifree, then $w-c t<2$.

(4) If $S$ possesses an essential $n$-gon for $n \geq 2$, then $w-c t \leq 2 \cos (\pi / n)$.

Note the following immediate corollary:

Corollary 1.8 If a quasi-Fuchsian surface $S$ possesses an essential 3-gon, then $1-c t \leq w \leq 1+c t$. 
Although we do not explicitly address this here, the results of Theorem 1.6(2), and Theorem 1.7(3) and (4) also hold for immersed surfaces. There is also a generalization of Theorem 1.6(3) to quasi-Fuchsian surfaces that yields a lower bound on $q$ in terms of $w$ and $c t$. However, due to its technical nature, it is not included.

In the final section we prove the following theorem in order to provide a source of examples. The method of proof was suggested by Ian Agol.

Theorem 1.9 Let $K$ be an alternating knot in the 3-sphere with hyperbolic complement. Let $S$ be a checkerboard surface obtained from a reduced alternating projection of $K$. Then $S$ is quasi-Fuchsian.

Corollary 1.10 A hyperbolic alternating knot or link possesses a quasi-Fuchsian surface with $w-c t \leq 1$.

Proof Every reduced projection of an alternating knot or link possesses 2-gon or 3-gon regions. By Theorem 1.7(4), the complementary checkerboard surface must satisfy $w-c t \leq 1$.

Corollary 1.11 A hyperbolic 2-bridge knot or link possesses a quasi-Fuchsian surface such that $w \leq c t$ and $c t \geq 0.5$.

Proof Any 2-bridge knot or link possesses a reduced alternating projection with at least one bigon. The complementary checkerboard surface is quasi-Fuchsian with an essential 2-gon. Thus $w-c t \leq 2 \cos (\pi / 2)=0$. With Theorem 1.7(1), this yields $2 c t \geq w+c t \geq 1$, and hence $c t \geq 0.5$.

Example 1.12 (Totally geodesic surfaces) In the standard alternating projection of the Borromean rings, each of the two checkerboard surfaces are quasi-Fuchsian with an essential 3-gon, so $1-c t \leq w \leq 1+c t$, where $w$ is the balanced width. In fact, the methods of Adams-Schoenfeld [2] imply these surfaces are totally geodesic (or this can be seen directly from the decomposition of the Borromean rings complement into ideal regular right-angled octahedra). Hence $c t=0$ and $w=1$. Note that in this case, for the balanced cusps, $q=\sqrt{2}$. Hence, Theorem 1.6(3) cannot be improved.

Similarly, we can consider the alternating links created from the 1-skeleton of the cubeoctahedron and the icosidodecahedron by replacing each vertex with a crossing in an alternating manner. In each case, the methods of [2] imply that both checkerboard surfaces are totally geodesic with $c t=0$, and such that they intersect each other at right angles. For the first link, the first of these surfaces possesses an essential 3-gon 
and therefore $w=1$ for it. The second surface has an essential 4-gon which lies on the first surface and one can then see, by symmetry of this 4-gon in the link complement that $w=\sqrt{2}$ for the second surface. For the second link, one surface again has an essential 3-gon and so $w=1$ for it. The second surface has an essential 5-gon and $w=(1+\sqrt{5}) / 2$.

Example 1.13 (The knot $8_{18}$ ) In the usual projection of the knot $8_{18}$, both checkerboard surfaces are nonorientable with no essential bigons in their complements. The surfaces have slopes \pm 8 . In each case, one can calculate from Jeffrey Weeks's computer program SnapPea [17] that the width of the surface is approximately 0.192655592 . Therefore in each case, ct must be at least 0.81 . In fact, there is a symmetry of the knot that takes one surface to the other, so they both have the same cusp thickness.

In Freedman-Freedman [10], the authors introduced the idea of attaching immersed annuli to the boundaries of existing surfaces to obtain closed essential immersed surfaces that lift to embedded surfaces in finite covers. In Cooper-Long-Reid [7], this type of construction was utilized to show that a compact connected irreducible 3-manifold with infinite fundamental group and incompressible boundary must either be covered by $F \times I$ for some surface $F$ or it must contain a closed immersed essential surface that lifts to am embedded surface in some $n$-fold cover. In the case of a cusped finite volume hyperbolic 3-manifold $M$ with a properly embedded orientable quasi-Fuchsian surface $S$ of width $w$ and cusp thickness $c t$, the preceding theorems yield this same result, since there exists a positive integer $n$ such that $n w-c t \geq 2$. Hence in the corresponding $n$-fold cover $M^{\prime}$ obtained by cutting $M$ open along $S$, and gluing together $n$ copies of the resulting manifold, annuli can be glued to the boundary of lifts of $S$ so that the resulting embedded closed surface is incompressible and its projection to $M$ is essential. In the following theorem, we determine bounds for $n$ when $c t<1$.

Theorem 1.14 Let $M$ be a cusped hyperbolic manifold with quasi-Fuchsian surface $S$ of width $w$ and cusp thickness $c t$. If for any integer $n>1$, ct $\leq(n-2) /(n+1)$, then $M$ contains a closed immersed essential surface $S^{\prime \prime}$ with $n-1$ order two disjoint closed singular curves. When $S$ is orientable, $S^{\prime \prime}$ lifts to an embedded surface in an $n$-fold cover.

Note that in the case $S$ is totally geodesic, the 2 -fold cover suffices.

Acknowledgements Thanks to Ian Agol, Joseph Masters and Alan Reid for their generous help. 


\section{Proofs}

The existence of an essential $n$-gon for a Fuchsian or quasi-Fuchsian surface implies that in the universal cover, there is a cyclic collection of circles or quasi-circles in the limit set of the pre-image of the surface, such that each one shares a point with the next, where each of these shared points corresponds to a parabolic fixed point for $\Gamma$.

In several of the subsequent proofs, we will take advantage of the following simple geometric lemma.

Lemma 2.1 If $H_{1}$ and $H_{2}$ are horocycles with disjoint interiors centered at two of the vertices of an ideal triangle $T$ as in Figure 1, and $a_{1}$ and $a_{2}$ are the lengths of $H_{1} \cap T$ and $H_{2} \cap T$ respectively, then $a_{1} \leq 1 / a_{2}$ with equality when the two horocycles are tangent.

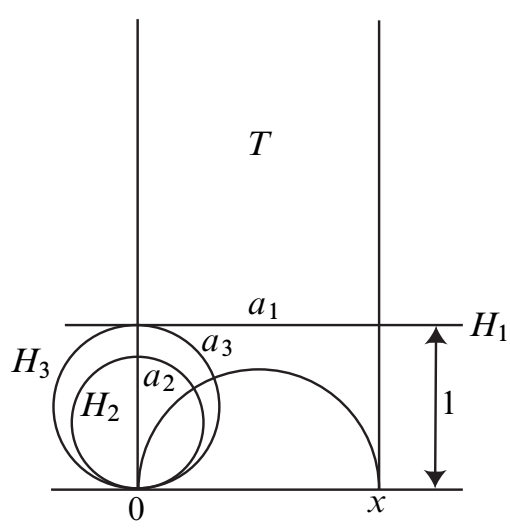

Figure 1: Distance on the tangent horocycles are reciprocals.

Proof For convenience, $H_{1}$ has been normalized to have height 1. From the figure, it follows that $a_{1}=x$. Reflecting the picture across a semicircular geodesic with endpoints at $x$ and $-x$, the horocycle $H_{3}$ centered at 0 is sent to a horocycle centered at $\infty$ with Euclidean height $x^{2}$. The arc $H_{3} \cap T$ is sent to a horizontal line segment in this horocycle of Euclidean length $x$ and height $x^{2}$. Hence, it has hyperbolic length $a_{3}=x / x^{2}=1 / x$. It is at least as large as $a_{2}$.

Proof of Theorem 1.6(1) An essential 2-gon implies that two quasi-circles share two distinct parabolic fixed points. Since in this case the surface is Fuchsian, the quasi-circles are actual circles, which, because the surface is embedded, cannot cross one another. Therefore, they must be identical, a contradiction. 
Proof of Theorem 1.6(2) Given an essential $n$-gon, $S$ lifts to a collection of geodesic planes, the limit sets of which contain a cyclic collection of $n$ circles, meeting consecutively at parabolic fixed points. Choose one of the parabolic fixed points to be at $\infty$. Two geodesic planes covering the surface are vertical half-planes with boundary lines $L_{1}$ and $L_{2}$ that are connected by a sequence of $n-2$ pairwise tangent circles $C_{1}, \ldots, C_{n-2}$. The distance between the two vertical planes in the corresponding horizontal horosphere $H$ (normalized to have height 1 ) is $w$. This distance is also bounded by the sum of the diameters of the circles between them. The diameter of each successive circle is determined by the diameter of the previous circle, $w$ and the size of the horosphere at the tangency point corresponding to the cusps. If the centers of the circles are not all in a line perpendicular to the boundaries of the two vertical planes, we can slide $L_{2}$ away from $L_{1}$ while maintaining the size and tangencies of the circles, and the size of the horospheres at each point of tangency until the chain of circles becomes taut, all centered along a line. Then it is still the case that the distance on each spherical horosphere between the two geodesic planes that share its limit point is exactly $w$. However, the distance on the horizontal horosphere between the two vertical planes has increased to a value we call $z$. Hence, from now on, we assume that the centers of all of the circles are in a line, and we need only consider what occurs for a chain of geodesics sharing endpoints (an ideal $n$-gon) with horocycles at those endpoints in the hyperbolic plane.

As in Figure 2, we decompose the $n$-gon $N$ into $n-2$ ideal triangles, and label the horocyclic lengths as shown. Note that $b_{i}+b_{i}^{\prime}=w$. From Lemma 2.1, we have that $w \leq 1 / b_{1}, b_{i+1} \leq 1 / b_{i}^{\prime}$ and $b_{n-3}^{\prime} \leq 1 / w$. Hence, we obtain:

$$
w \leq \frac{1}{b_{1}}=\frac{1}{w-b_{1}^{\prime}} \leq \frac{1}{w-\frac{1}{b_{2}}} \leq \cdots \leq \frac{1}{w-\frac{1}{w-\ldots \frac{1}{w-1 / w}}}
$$

We are interested in values of $w$ where the function

$$
f_{k}(w)=\frac{1}{w-\frac{1}{w-\ldots \frac{1}{w-1 / w}}}-w
$$

is nonnegative. The function $f_{k}(w)$ can be seen to be decreasing since $f_{1}(w)=1 / w$ and $f_{n}(w)=1 /\left(w-f_{n-1}(w)\right)$ then has negative derivative. Since $f_{k}(w)$ becomes negative for large enough $w$, the largest value for $w$ that satisfies the inequality occurs when the inequality becomes an equality. This happens when each horocycle is tangent to the two adjacent horocycles. Since $z \geq w$, the maximum $w$ occurs when $z=w$ and the horizontal horosphere is tangent to the two horospheres on the end. This case occurs for a regular horocyclic $n$-gon with tangent horocyclic edges of length $w$. Elementary 


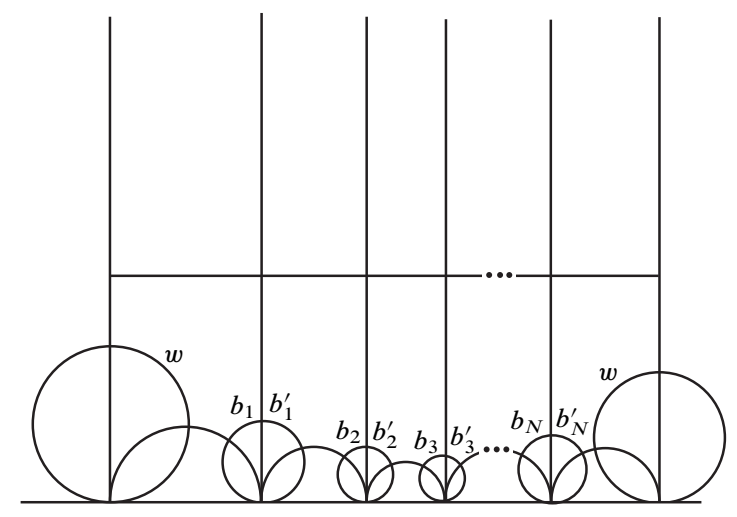

Figure 2: Decompose the $n$-gon into $N$ ideal triangles, where $N=n-3$.

hyperbolic geometry can then be used to show that the length of the horocyclic edges is given by $w=2 \cos (\pi / n)$.

Proof of Theorem 1.6(3) Choose $H$ to be a horosphere covering the cusp boundary, centered at $\infty$, normalized to have Euclidean height 1 and let $A$ be another horosphere covering the cusp boundary tangent to $H$ and with center $x$.

In the case that the point of tangency is not in the surface, There must exist two hemispheres covering $S$, the boundaries of which are two circles both passing through $x$, and neither circle containing the other. The distance between these planes on $A$ must be $w$. Lemma 2.1 implies that if the diameter of one is $d$, the diameter of the other is $1 /(w-1 / d)$. There are also parallel vertical planes covering $S$ spaced a Euclidean distance $w$ apart. None of these can intersect either of the hemispheres other than on their boundary. In particular, the diameters of the two hemispheres must both be at most $w$. The smallest possible value of $w$ for this to be true occurs when the two hemispheres have equal diameter, which corresponds to $d=2 / w$. Then the smallest $w$ occurs when the hemispheres each touch two vertical planes separated by a distance of $w$, so $2 / w=w$ and we find $w \geq \sqrt{2}$. The length $q$ must always be at least as large as $w$.

In the case $q=\sqrt{2}$, then $q=w$ and both hemispheres touch each of the vertical planes. Hence the point of contact of one hemisphere with one vertical plane must be a parabolic fixed point $y$ and the distance on the corresponding horosphere $H^{\prime}$ between the vertical plane and the hemisphere must be exactly $w=\sqrt{2}$. However, since $H$ has height 1 and the diameter of the hemisphere is $\sqrt{2}$, the distance on $H^{\prime}$ between 
the two planes is at most $1 / \sqrt{2}$, a contradiction. So when the point of tangency is not in the surface, $q>\sqrt{2}$.

In the case that the point of tangency is in the surface,there exists a vertical plane $P$ covering $S$ with boundary line containing $x$. Then there must exist hemispheres $P_{1}$ and $P_{2}$ covering $\mathrm{S}$ of diameter $1 / w$ to either side of $P$, both tangent to $P$ at $x$. The parabolic isometry $T$ corresponding to $q$ must translate the xy-plane a distance $w$ perpendicular to $P$ and a distance $e$ parallel to $P$, as in Figure 3. So $q^{2}=w^{2}+e^{2}$.

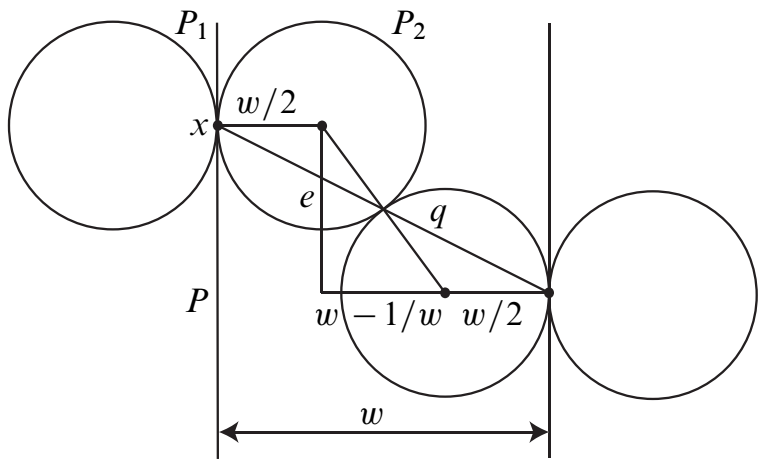

Figure 3: Proving $q \geq \sqrt{2}$

There are two possibilities. First, $T\left(P_{1}\right)$ is $P_{2}$. In this case, there are parabolic isometries identifying $P$ to $T(P), P$ to $P_{2}$ and $P_{2}$ to $T(P)$. These force an ideal edge in the manifold to be identified to itself with reverse orientation, generating an isometry in $\Gamma$ with a fixed point, a contradiction.

Second, $T\left(P_{1}\right)$ does not overlap $P_{2}$ in its interior. So if $w<\sqrt{2}, e>0$ to prevent the two hemispheres from overlapping. As in Figure 3, we see that

$$
\frac{1}{w^{2}} \leq e^{2}+\left(w-\frac{1}{w}\right)^{2}
$$

Hence $e^{2} \geq 2-w^{2}$, and $q \geq \sqrt{2}$.

In the case $q=\sqrt{2}$, the planes $P_{2}$ and $T\left(P_{1}\right)$ touch one another. This produces an obvious essential 4-gon that is the projection of the 4-gon with boundary contained in four horospheres and the cyclic collection of four pairwise tangent totally geodesic planes appearing in Figure 3.

Note that the case $q=\sqrt{2}$ is realized by the balanced cusps for the Borromean rings complement. 
Proof of Theorem 1.7(1) Suppose $w+c t \leq 1$. Choose $H$ to be a horosphere covering the cusp boundary, centered at $\infty$, normalized to have Euclidean height 1 and let $A$ be another horosphere covering the cusp boundary tangent to $H$ and with center $x$.

Suppose first that the center of $A$ does not lie on a quasi-line for $S$. Note that any disk of diameter $w+c t$ on a horosphere covering a cusp must intersect both sandwiching planes for some quasi-circle passing through its center. Since the disk on $A$ of diameter $w+c t$ centered at the point of tangency must intersect a pair of planes $P_{1}$ and $P_{2}$ sandwiching a limiting quasi-circle $Q$, Lemma 2.1 implies the diameter of each of these hemispheres is at least 1 . Hence each hemisphere must intersect a pair of vertical planes sandwiching a quasi-line $L$. One of the boundaries of the two circles lies inside the disk bounded by $Q$. This forces $Q$ to cross $L$, contradicting the fact the surface is embedded.

Suppose now that the center of $A$ does lie on a quasi-line $L_{0}$ corresponding to $S$. Let $P$ be one of the two geodesic planes through $x$ that sandwich $L_{0}$. Then there exists a hemisphere $P^{\prime}$ sandwiching a quasi-circle $Q$ through $x$ that is a distance $w$ on $A$ from $P$ such that the circle bounding $P^{\prime}$ is contained in the disk bounded by $Q$. The diameter of $P^{\prime}$ is the smallest possible when $P$ is a vertical plane. In this case, the diameter of $P^{\prime}$ is exactly $1 /(w+c t) \geq 1$. Since the point $x$ is between two vertical planes sandwiching $L_{0}$ which themselves are a distance $c t$ apart, and which are a distance $w-c t$ from the first of another such pair of vertical planes $P_{1}^{\prime \prime}$ and $P_{2}^{\prime \prime}$ sandwiching a quasi-line $L_{1}$, the plane $P^{\prime}$ will intersect both of $P_{1}^{\prime \prime}$ and $P_{2}^{\prime \prime}$. If $w+c t<1$, then $Q$ is forced to cross $L_{1}$ contradicting the fact $S$ is embedded. Hence, the only possibility is that $Q$ just touches each of $L_{1}$ and $L_{0}$ at points that are also touching the outside vertical sandwiching planes and $w+c t=1$.

Proof of Theorem 1.7(2) As in the proof of the previous result, if $w+c t=1$, it must be the case that two quasi-lines $L_{0}$ and $L_{1}$ both touch a quasi-circle $Q$ at parabolic fixed points. Hence there is a 3 -gon with edges in the corresponding three topological planes and in the three horospheres corresponding to the parabolic fixed points they share. This projects to an essential 3-gon in the manifold.

Proof of Theorem 1.7(3) Let $D$ be an essential $n$-gon that compresses the boundary of $M-N(S)$. The boundary of $D$ consists of $2 n$ arcs, alternating between lying in $\partial M$ and lying in $S$. Note that $n \geq 2$ by the boundary-incompressibility of $S$. Lift $D$ to a disk $D^{\prime}$ in $H^{3}$ so that one of the horospheres that intersects $\partial D^{\prime}$ is centered at $\infty$. Then in the chain of horospheres intersecting $\partial D^{\prime}$, there must be a smallest one, or at the very least one that is as small as its neighbors and smaller than one of its neighbors. Call this horosphere $A$ and its two neighbors $A^{\prime}$ and $A^{\prime \prime}$. Let $d$ be the 
Euclidean diameter of $A$. The distance from the center of $A$ to the centers of each of $A^{\prime}$ and $A^{\prime \prime}$ must be at least $d$ and greater than $d$ for one of them, say for $A^{\prime \prime}$. This is because the corresponding three horoballs must have disjoint interiors and if one of them had center closer than $d$ to the center of $A$, it would have to be smaller than $A$ to avoid overlapping it on its interior.

The centers of $A^{\prime}$ and $A^{\prime \prime}$ must lie on quasi-circles $L^{\prime}$ and $L^{\prime \prime}$ which are limit sets of topological planes covering $S$ such that each of $L^{\prime}$ and $L^{\prime \prime}$ pass through the center of $A$. That they lie on different quasi-circles follows from the fact that $n$ is minimal.

There are geodesic planes with limiting circles passing through the center of $A$, two sandwiching $L^{\prime}$, call them $P_{1}^{\prime}$ and $P_{2}^{\prime}$ and two sandwiching $L^{\prime \prime}$, call them $P_{1}^{\prime \prime}$ and $P_{2}^{\prime \prime}$. The distance on $A$ between the planes in a pair must be $v$, where $v \leq c t$. Let $P_{2}^{\prime}$ and $P_{2}^{\prime \prime}$ be the two planes, one from each pair, that have largest diameter.

The fact that the center of $A$ must be a distance at least $d$ from the center of $A^{\prime}$ and more than $d$ from $A^{\prime \prime}$ implies that the diameter of $P_{2}^{\prime}$ must be at least $d$ and the diameter of $P_{2}^{\prime \prime}$ must be greater than $d$. This implies that the distance on $A$ from $P_{2}^{\prime}$ to the peak of $A$ is at most 1 and the distance on $A$ from $P_{2}^{\prime \prime}$ to the peak of $A$ is less than 1 .

If one of the hemispheres is contained in the half-space bounded by the other, then the distance between them on $A$ is less than 1 . This implies $w+c t<1$, and therefore $w-c t<2$. On the other hand, if neither hemisphere is contained in the half-space bounded by the other, the distance on $A$ between these two planes is less than 2 . But in this case, the distance between them on $A$ is exactly $w-v$, yielding $w-v<2$. This implies $w-c t<2$, as we wished to show.

Proof of Theorem 1.7(4) Let $D$ be an essential $n$-gon that compresses the boundary of $M-N(S)$. Lift $D$ to a disk $D^{\prime}$ in $H^{3}$ so that one of the horospheres that intersects $\partial D^{\prime}$, call it $H_{0}$, is centered at $\infty$. Then $\partial D^{\prime}$ consists of $2 n$ arcs, alternating between a cyclic sequence of horospheres covering the cusps and a cyclic sequence of topological planes covering the surface, with limit sets of subsequent planes sharing parabolic fixed points corresponding to the horospheres. Let $L_{0}$ and $L_{n-1}$ be the quasi-lines that are the limit sets of the two topological planes that share the parabolic fixed point at $\infty$. Let $L_{1}, \ldots, L_{n-2}$ be the quasi-circles that are the limit sets of the chain of topological planes. Then $L_{i}$ shares a parabolic fixed point $x_{i+1}$ with $L_{i+1}$, where $x_{i}$ is the center of the horosphere $H_{i}$ covering the cusps. Corresponding to the choice of a parabolic fixed point $x$ and limit set $L_{i}$ containing that point, there are two sandwiching geodesic planes with limit sets passing through $x$ that yield the cusp thickness. For $x_{i}$ and $L_{i}$, let $P_{i}^{O}$ be the outer plane with circle limit set containing $L_{i}$ and $P_{i}^{I}$ be the inner 
plane with limit set contained inside the topological disk bounded by $L_{i}$. For $x_{i+1}$ and $L_{i}$, define the outer plane to be $Q_{i}^{O}$ and the inner plane to be $Q_{i}^{I}$. In the case of $x_{0}=\infty$, define $P_{0}^{O}, P_{0}^{I}, Q_{n-1}^{O}$ and $Q_{n-1}^{I}$ so that $P_{0}^{O}$ and $Q_{n-1}^{O}$ are adjacent in $H$. See Figure 4.

Note that the distance on $H_{i}$ between $Q_{i}^{O}$ and $P_{i+1}^{O}$ is exactly $w-c t$.

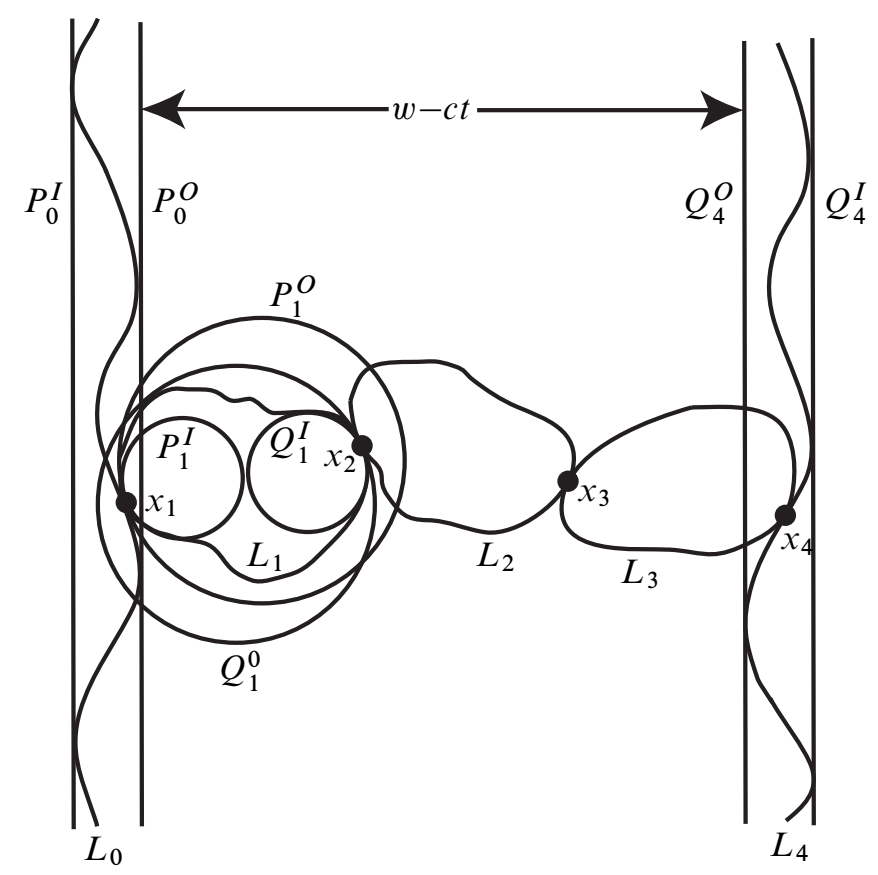

Figure 4: Lifting an essential $n$-gon for a quasi-Fuchsian surface

Note also that the disks bounded by $P_{i}^{O}$ and $Q_{i}^{O}$ both contain $L_{i}$. As in the proof of Theorem 1.6(3), we will move all the points of tangency between the geodesic planes to lie in a line perpendicular to the boundary lines of $P_{0}^{O}$ and $Q_{n-1}^{O}$, without changing the sizes of the geodesic planes or the horospheres. To do so, suppose we have already lined up $x_{1}, \ldots, x_{i-1}$. Then $x_{i}$ lies on $\partial P_{i}^{O}$, and inside $\partial Q_{i}^{O}$. Similarly, $x_{i+1}$ lies on $\partial Q_{i}^{O}$, and inside $\partial P_{i}^{O}$. The point $x_{i}$ will remain fixed, but otherwise, we will rotate $\partial P_{i}^{O}$ and all of the circles, tangency points and horospheres corresponding to indices greater than $i$, so that their relation to one another does not change, but so that a diameter of $\partial Q_{i}^{O}$ intersects $x_{i}$. Now, fixing the position of $\partial Q_{i}^{O}$, we rotate the tangency point $x_{i+1}$ around $\partial Q_{i}^{O}$, pulling along $\partial P_{i+1}^{O}, H_{i+1}$ and all of the circles, horospheres and tangency points with indices greater than $i+1$. Repeating this process, we have ultimately moved $P_{0}^{O}$ and $Q_{n-1}^{O}$ further apart and lined up all of the tangency points on a line perpendicular to both, while preserving the sizes of the 
horospheres and sandwiching geodesic planes. Note that we no longer respect the limit sets of the preimages of the original quasi-Fuchsian surface in this process. Let the new distance between $P_{0}^{O}$ and $Q_{n-1}^{O}$ on $H$ be denoted $z$, which is at least $w-c t$. An upper bound for $z$ is realized when the fixed points for the horospheres occur at the farthest possible point to the right, which is on the boundary of $P_{i}^{O}$. However, if $\partial Q_{i}^{O}$ is greater than $\partial P_{i}^{O}$,then $\partial P_{i+1}^{O}$ will be smaller than it could be. Hence, for maximal $z$, it must be that $P_{i}^{O}=Q_{i}^{O}$. Then, exactly as in the proof of Theorem 1.6, but with $w-c t$ replacing $w$, we see that $w-c t \leq 2 \cos (\pi / n)$.

\section{Surfaces with multiple boundaries on a cusp}

Let $S$ be a quasi-Fuchsian surface with multiple boundary components on a cusp $C$. Let $H$ be a horosphere covering $C$ centered at $\infty$ in the upper-half-space model of $H^{3}$. The surface $S$ lifts to a collection of topological planes. Certain of those planes have $\infty$ as a limit point, so their limit sets appear as quasi-lines in the xy-plane. Let $L_{1}$ and $L_{2}$ be two quasi-lines separated by no others. Each of $L_{1}$ and $L_{2}$ are sandwiched by vertical geodesic planes $P_{1}^{\prime}, P_{1}^{\prime \prime}$ and $P_{2}^{\prime}, P_{2}^{\prime \prime}$, each pair yielding the respective cusp thickness of the quasi-line. Define $P_{1}$ and $P_{2}$ to be the two planes equidistant between $P_{1}^{\prime}, P_{1}^{\prime \prime}$ and $P_{2}^{\prime}, P_{2}^{\prime \prime}$ respectively. Define the relative width of $L_{1}$ and $L_{2}$ to be the distance in $H$ between $P_{1}$ and $P_{2}$. Define the minimum (maximum) width in this cusp to be the minimum (maximum) of the relative width over all pairs of adjacent quasi-lines corresponding to this cusp. If there are boundary components on more than one cusp, choose cusp sizes so that the minimum (maximum) widths match and then expand the cusps until two cusps touch or until one cusp touches itself. Define the balanced minimum (maximum) width of the surface to be the resulting minimum (maximum) width. We denote the balanced minimum width by $w_{\min }$ and the balanced maximum width by $w_{\max }$. Note that in the case there is only one boundary component on a cusp, the balanced minimum and maximum widths revert to the balanced width previously defined.

In the case that the surface is separating, denote each of the resulting components by I and II. There must be an even number of boundary components on each cusp and we can define, as above, both a minimum and maximum width for each component. These are denoted $w_{\min }^{\mathrm{I}}, w_{\max }^{\mathrm{I}}, w_{\min }^{\mathrm{II}}, w_{\max }^{\mathrm{II}}$.

Define the cusp thickness of the surface to be the maximum of the cusp thickness over all boundary components.

The proofs for the following theorem are a direct generalization of the proofs of Theorem 1.7. 
Theorem 3.1 Let $S$ be a quasi-Fuchsian surface properly embedded in a closed 3manifold $M$, the interior of which is a cusped hyperbolic 3-manifold, such that $S$ has more than one boundary component on at least one cusp.

(1) $w_{\max }+c t \geq 1$.

(2) If $S$ is semifree, then $w_{\min }-c t<2$.

(3) If $S$ possesses an essential $n$-gon for $n \geq 2$, then $w_{\min }-c t \leq 2 \cos (\pi / n)$.

In the case $S$ separates, the results hold for the $w_{\max }$ and $w_{\min }$ defined to each side.

Example 3.2 Let $\mathrm{M}$ be a noncompact hyperbolic 3-manifold containing an embedded quasi-Fuchsian quadruply-punctured sphere or twice-punctured torus $S$. Then the hyperbolic structure on $S$ must respect certain symmetries of $S$ that permute the boundary components. (See Ruberman [14].) In particular, this implies that the boundary components of $S$ all have the same cusp thickness.

In the case of a twice-punctured torus in a knot complement with meridional boundary components, it will be the case that $w_{\min }^{\mathrm{I}}=w_{\max }^{\mathrm{I}}=w^{\mathrm{I}}$ and $w_{\min }^{\mathrm{II}}=w_{\max }^{\mathrm{II}}=w^{\mathrm{II}}$ since the cusp boundary is only cut into two pieces. For the quadruply-punctured sphere, it is possible to have four different max and min width values.

\section{Checkerboard surfaces}

In this section, we prove that a checkerboard surface generated by a reduced alternating knot or link diagram for a hyperbolic knot or link is always quasi-Fuchsian. Hence the results of Theorem 1.7 apply to such a surface.

Note that work of Menasco [12] shows that a nontrivial alternating knot or link has hyperbolic complement if and only if it is neither obviously composite in any alternating diagram, nor an obvious 2-braid in its alternating diagram. Hence, one can immediately determine whether or not a given alternating knot or link is hyperbolic.

Proof of Theorem 1.9 A method for decomposing a knot or link complement into octahedra placed between the crossings was introduced by Dylan Thurston. It is described in detail in Yokota [18]. Each octahedron has two ideal vertices and four nonideal vertices. Truncating the octahedra at their ideal vertices and splitting each along a square through the four nonideal vertices yields a decomposition of the link exterior $M$ into a collection of cubes. For a reduced alternating projection, this collection satisfies the necessary conditions to be a nonpositively curved cubing (denoted $\mathrm{np}$ 
cubing) as in Aitchison-Rubenstein [3]. Take the collection of two squares in each cube, each of which bisects the cube, and is parallel to a pair of opposite faces and intersects one of the toroidal boundary components. Together these squares glue up to produce the two checkerboard surfaces, denoted $S_{1}$ and $S_{2}$.

Double the manifold across the toroidal boundary components. The resulting manifold $M^{\prime}$ inherits an np cubing, and $S_{1}$ and $S_{2}$ double to closed surfaces $S_{1}^{\prime}$ and $S_{2}^{\prime}$ that are totally geodesic in the new np cubing. Hence, in particular, $S_{1}^{\prime}$ and $S_{2}^{\prime}$ must be $\pi_{1}$-injective in $M^{\prime}$. This implies That $S_{1}$ and $S_{2}$ are $\pi_{1}$-injective in $M$ as well.

If either $S_{1}$ or $S_{2}$ is accidental, a nontrivial nonboundary parallel curve on it is homotopic into the boundary torus. The doubled surface then has two distinct nontrivial curves homotopic to one another, contradicting the fact it is $\pi_{1}$-injective in $M^{\prime}$. So each checkerboard surface must be either quasi-Fuchsian or a virtual fiber. Suppose for $S_{1}$ it is the latter. Then $S_{1}^{\prime}$ is a virtual fiber in $M^{\prime}$. Hence it lifts to a fiber $S_{1}^{\prime \prime}$ in a finite cover $M^{\prime \prime}$. The manifold $M^{\prime \prime}$ inherits an np cubing as well, and $S_{1}^{\prime \prime}$ consists of a collection of squares bisecting the cubes in this cubing. Note that the essential tori across which we doubled to create $M^{\prime}$ lift to a finite collection of essential tori in $M^{\prime \prime}$, cutting $M^{\prime \prime}$ into pieces, each of which has a hyperbolic metric of finite volume.

As in [3], let $C$ be the union of all the line segments in the squares that bisect the square and are parallel to the edges. The union of $\mathrm{C}$ is a collection of immersed geodesics in the polyhedral metric.

Cutting $M^{\prime \prime}$ open along $S_{1}^{\prime \prime}$ yields $S_{1}^{\prime \prime} \times I$. The collection of immersed geodesics formed by $C$ appear on both $S_{1} \times 0$ and $S_{1} \times 1$. They can be extended into the interior of $S_{1}^{\prime \prime} \times I$ by taking the squares bisecting cubes in the cubing of $S_{1}^{\prime \prime} \times I$ that have boundary in these geodesics and then continuing to glue on squares until finished.

As in [3], one can show the result is homeomorphic to $C \times I$ and intersects both $S_{1}^{\prime \prime} \times 0$ and $S_{1}^{\prime \prime} \times 1$ in $C$. Hence $M^{\prime \prime}$ must be a fiber bundle with periodic monodromy. Thus, as in Scott [15], it has either Euclidean or $H^{2} \times R$ geometry, contradicting the fact it is cut into a finite number of hyperbolic pieces by a finite collection of essential tori.

\section{Applications}

Proof of Theorem 1.14 Theorem 1.7(1) says $w+c t \geq 1$. Since $c t \leq(n-2) /(n+1)$, $w \geq 1-(n-2) /(n+1)=3 /(n+1)$ and hence $n w-c t \geq 2$.

Let $S^{\prime}$ be the orientable surface that forms the boundary of a neighborhood of the surface $S$. Take an annulus with one boundary component shared with one of the 
boundaries of $S^{\prime}$ and the other shared with the other boundary component of $S^{\prime}$ such that the annulus wraps around the cusp boundary $n$ times, intersecting itself along $n-1$ disjoint singular curves. Call the resulting closed immersed surface $S^{\prime \prime}$. In the universal cover $H^{3}, S^{\prime \prime}$ is covered by a surface obtained as follows. Take the collection of topological planes that covered $S^{\prime}$, and remove from each its intersections with the interiors of horoballs covering the cusps. The resulting boundaries are then connected in pairs by disks on the horospheres that cover the annuli, each of which has two ideal points at the center of the horosphere. The surface $S^{\prime \prime}$ compresses if and only if a quasi-Fuchsian surface with width $n w$ and cusp thickness $c t$ compresses. But since $n w-c t \geq 2$, that surface must be totally knotted by Theorem 1.7(3). So a connected lift of $S^{\prime \prime}$ cannot have a compressing disk. Hence, $S^{\prime \prime}$ is $\pi_{1}$-injective. The surface obviously lifts to an embedding in the $n$-fold cover when $S$ is orientable.

\section{References}

[1] C Adams, H Bennett, C Davis, M Jennings, J Novak, N Perry, E Schoenfeld, Totally geodesic Seifert surfaces in hyperbolic knot complements II, submitted for publication

[2] C Adams, E Schoenfeld, Totally geodesic Seifert surfaces in hyperbolic knot complements $I$, to appear in Geometriae Dedicata

[3] I R Aitchison, J H Rubinstein, An introduction to polyhedral metrics of nonpositive curvature on 3-manifolds, from: "Geometry of low-dimensional manifolds, 2 (Durham, 1989)", London Math. Soc. Lecture Note Ser. 151, Cambridge Univ. Press, Cambridge (1990) 127-161 MR1171913

[4] F Bonahon, Bouts des variétés hyperboliques de dimension 3, Ann. of Math. (2) 124 (1986) 71-158 MR847953

[5] R D Canary, D B A Epstein, P Green, Notes on notes of Thurston, from: "Analytical and geometric aspects of hyperbolic space (Coventry/Durham, 1984)", London Math. Soc. Lecture Note Ser. 111, Cambridge Univ. Press, Cambridge (1987) 3-92 MR903850

[6] D Cooper, D D Long, Some surface subgroups survive surgery, Geom. Topol. 5 (2001) 347-367 MR1825666

[7] D Cooper, D D Long, A W Reid, Essential closed surfaces in bounded 3-manifolds, J. Amer. Math. Soc. 10 (1997) 553-563 MR1431827

[8] R Crowell, Genus of alternating link types, Ann. of Math. (2) 69 (1959) 258-275 MR0099665

[9] S R Fenley, Quasi-Fuchsian Seifert surfaces, Math. Z. 228 (1998) 221-227 MR1630563 
[10] B Freedman, M H Freedman, Kneser-Haken finiteness for bounded 3-manifolds locally free groups, and cyclic covers, Topology 37 (1998) 133-147 MR1480882

[11] D Gabai, Genera of the alternating links, Duke Math. J. 53 (1986) 677-681 MR860665

[12] W Menasco, Closed incompressible surfaces in alternating knot and link complements, Topology 23 (1984) 37-44 MR721450

[13] K Murasugi, On the genus of the alternating knot. I, II, J. Math. Soc. Japan 10 (1958) 94-105, 235-248 MR0099664

[14] D Ruberman, Mutation and volumes of knots in $S^{3}$, Invent. Math. 90 (1987) 189-215 MR906585

[15] P Scott, The geometries of 3-manifolds, Bull. London Math. Soc. 15 (1983) 401-487 MR705527

[16] W P Thurston, The Geometry and Topology of Three-Manifolds, Princeton Univ. Math. Dept. Notes (1979)

[17] J Weeks, SnapPea Available at http://www.geometrygames.org/SnapPea/

[18] Y Yokota, On the volume conjecture for hyperbolic knots arXiv:math.QA/0009165

Department of Mathematics and Statistics, Williams College

Williamstown, MA 01267

Colin. C.Adams@williams .edu

Received: 10 October 2006

Algebraic 83 Geometric Topology, Volume 7 (2007) 